displayed in traditional PDF format and DECIDE A and B formats. Throughout the lecture participants will answer questions with 'Clickers' and be randomly assigned to alternative presentation formats by concealed allocation and blinding, through the use of eyepatches.

Results We will present results from the trial at the conference. Discussion If our approach of integrating randomised trials into educational sessions is feasible and provides valid results we will conduct multiple such trials in DECIDE.

Implications for Guideline Developers and Users Optimised GL presentation formats and sufficient conceptual understanding, as researched in this trial, should facilitate the uptake of trustworthy CPG and application of research evidence in practice.

\section{CLINICAL PRACTICE GUIDELINES FOR AUSTRALIAN GENERAL PRACTITIONERS: HOW IMPLEMENTABLE ARE THEY?}

S Chakraborty, D Mazza. Department of General Practice, School of Primary Health Care, Monash University, Melbourne, Australia

\section{0:1136/bmjgs-2013-002293.60}

Background Many guidelines have been published that are relevant to Australian general practitioners. However, it is unclear whether these guidelines have the attributes required for facilitating implementation.

Objectives To determine the proportion of current Australian general practice guidelines that have incorporated the attributes required for facilitating implementation.

Methods We conducted an audit of the National Health and Medical Research Council Clinical Guidelines Portal to identify guidelines published between 2007 and 2011 that listed general practitioners (GPs) as a primary user and examined them for attributes identified in literature as facilitating implementation.

Results A total of 146 guidelines targeting Australian GPs were identified in our study. Approximately 46\% of these guidelines were developed by "collaborating authors", with $27 \%$ and $19 \%$ developed by "government organisations" and "not-for-profit organisations", respectively. Almost half $(43 \%)$ of the guidelines did not state the methodology used, with 33\% using "expert opinion" and only $16 \%$ using "systematic literature reviews". Only $14 \%$ of the guidelines were endorsed by professional colleges and only $10 \%$ of the guidelines were governmentapproved. Additional resources to facilitate guideline uptake were included for only $23 \%$ of the guidelines.

Discussion While some attributes of implementation have been incorporated into general practice guidelines, many are absent from most of these guidelines. Given the rapid growth in evidence-based guidelines in Australia, it is imperative that clinical practice guidelines incorporate the attributes necessary for facilitating implementation.

Implications for Guideline Developers/Users Developing an evidence-based guideline implementability framework may be useful for improving the development and dissemination of guidelines.

\section{PRIMARY CARE PHYSICIANS' VIEWS ON RELEVANCE OF CLINICAL GUIDELINE RECOMMENDATIONS: DELPHI PANEL}

${ }^{1} \mathrm{~N}$ Steel, ${ }^{1} \mathrm{~A}$ Abdelhamid, ${ }^{2} \mathrm{~T}$ Stokes, ${ }^{1} \mathrm{R}$ Fleetcroft, ${ }^{3} \mathrm{~N}$ Qureshi, ${ }^{1} \mathrm{~A}$ Howe. ${ }^{1}$ Norwich Medical School, University of East Anglia, Norwich, UK; ${ }^{2}$ National Institute for Health and Clinical
Excellence (NICE), Manchester, UK; ${ }^{3}$ University of Nottingham, Nottingham, United Kingdom

\section{0:1136/bmjgs-2013-002293.61}

Background National clinical guideline developers, such as the UK's National Institute for Health and Clinical Excellence (NICE), produce high quality guidelines, yet primary care practitioners (PCPs) may question the relevance of the evidence and recommendations to a primary care (PC) population.

Objectives To evaluate PCPs' views about the relevance of NICE clinical guidelines to PC.

Methods An online Delphi panel of 28 PCPs, recruited regionally and nationally, reviewed 14 guideline recommendations: 8 supported by PC relevant evidence and 6 by evidence from elsewhere. Panellists scored recommendations twice, on a scale of 1-9 (9 = highly relevant for PC), before and then again after reading a summary of the evidence, including study setting and population. They also commented on factors influencing guideline validity and PC implementability.

Results 25 PCPs (89\%) completed the Delphi. Overall mean scores were 7.4 (range 6.2-8.2) before reading the evidence summary, and 6.6 (4.6-8.3) after. Mean scores for the 8 recommendations supported by PC evidence were 7.4 before and 7.2 after (change -0.2). Mean scores for the 6 with evidence from elsewhere were 7.4 before and 5.8 after (change -1.6). Factors perceived to influence implementation included clarity, brevity, and relevance to PC.

Discussion PCPs' ratings of PC guideline validity dropped when they became aware that substantial supporting evidence for the guidelines had come from non PC settings. The relevance of the evidence to PC patients was important.

Implications for Guideline Developers/Users Developers should explicitly describe the relevance of available evidence for PCPs and their patients.

\section{IF RAPID REVIEWS ARE THE ANSWER, WHAT IS THE QUESTION?}

$B$ Ireland. TheEvidenceDoc, USA

10:1136/bmjgs-2013-002293.62

Background The Institute of Medicine recommended standards for systematic review, but some guideline developers find the standards time and resource intensive. Rapid reviews are becoming a popular method to appraise and summarise evidence. But what are rapid reviews and do they replace or rely on systematic review?

Objectives To clarify major differences between rapid reviews and systematic reviews, especially aims, methods and uses for guidelines and policy.

Methods Overview of reviews and examination of organisational policies for rapid review focusing on reasons users request rapid review, methods used to produce them, and the uses of those syntheses.

Results There is no standards methodology for producing rapid review, nor is there consistency in intended use. Some organisations rely on systematic reviews to produce rapid review, while others incorporate short cuts in systematic review process. In addition to faster production, some users of rapid review are seeking product that is more clinically relevant and ready for implementation. 
Discussion There are many ways to speed up systematic review, some at risk of introducing bias. Without clear understanding of the reasons users request rapid review and their expectations for the evidence product, simply speeding the time frame may not address all of user needs for evidence they can implement quickly.

Implications for Guideline Developers/Users Until there is consensus on what the label rapid review describes, users will need to identify their own minimal standards and evaluate adherence. Developers should be clear about user expectations for using the evidence.

\section{RAPID GUIDELINES: A SYSTEMATIC REVIEW}

${ }^{1,2,3} \mathrm{M}$ Falavigna, ${ }^{4}$ I Ikobaltzeta, ${ }^{3} \mathrm{~F}$ Sakhia, ${ }^{3} \mathrm{Y}$ Zhang, ${ }^{3} \mathrm{~N}$ Santesso, ${ }^{5} \mathrm{~S}$ Norris, ${ }^{3,6} \mathrm{H}$ Schünemann. 'Universidade Federal do Rio Grande do Sul, Porto Alegre, Brazil; ${ }^{2}$ IATS - National Institute of Science and Technology for HTA, Porto Alegre, Brazil; ${ }^{3}$ Department of Clinical Epidemiology and Biostatistics. McMaster University, Hamilton, Canada; ${ }^{4}$ OSTEBA - Basque Office for Health Technology Assessment, Vitoria-Gasteiz, Spain; ${ }^{5}$ World Health Organization, Geneve, Switzerland Department of Medicine; ${ }^{6}$ McMaster University Hamilton, Canada

\section{0:1136/bmjgs-2013-002293.63}

Background Guidelines often take two or more years to be developed. This timeframe is not practical for providing guidance in situations when rapid advice is needed.

Objectives To describe current practices about the development of rapid guidelines and to provide advice about adequate methodology.

Methods We performed a systematic review, including grey literature, to identify (1) rapid guidelines, defined as guidelines produced in a shortened time frame, and (2) methodological manuals addressing its development.

Results We only documents by WHO and NICE that described methods and actual guidelines. The WHO handbook describes "rapid advice guidelines"; guidelines produced in response to a public health emergency in which WHO is required to provide rapid global leadership and guidance. This advice should be produced within 1 to 3 months and be evidence-informed, however, it may not be supported by full reviews of the evidence. We identified six WHO rapid guidelines and one methodological guidance paper based on a WHO guideline. NICE produces "short clinical guidelines"; guidelines that address only part of a care pathway, allowing rapid (11-13-month) development of guidance on aspects of care for which the NHS requires urgent advice. We identified 18 NICE short clinical guidelines.

Discussion Literature is lacking about rapid guidelines and the intended role appears to differ. Despite its relevance, there are few rapid guidelines published and clarity about the terminology is needed.

Implications for Guideline Developers We will provide a framework for those developing rapid guidelines, including practical advice and clarification about the terminology used.

\section{USING RAPID REVIEWS TO INFLUENCE GUIDANCE DEVELOPMENT IN THE EMERGENCY DEPARTMENT SETTING}

J Ramachandran. Kaiser Permanente, Southern California Permanente Medical Group, Pasadena, USA

10:1136/bmjqs-2013-002293.64
Background Decision making within health care organisations often requires rapid response to emergent, controversial or highimpact issues affecting clinical and operational practices.

Context CT imaging without oral contrast for patients admitted into the Emergency Department (ED) for abdominal/pelvic pain has been proposed as a viable option to reduce the risk of contrast-induced nephropathy and allergic reactions, as well as emergency room delays and overall length of stay (LOS) in the ED. A centralised evidence assessment unit within a large health care organisation was asked to conduct a rapid evidence review to inform the development of evidence-based guidance.

Description of Best Practice A 5 -step rapid review process was initiated, including: 1) Communicating with key stakeholders to determine relevant populations, interventions, comparisons, outcomes, timing and settings (PICOTS); 2) Conducting a comprehensive evidence search using a pre-established list of key databases and other sources to identify high-quality guidelines, systematic reviews and clinical trials evaluating the efficacy and diagnostic accuracy of conducting abdominal CT with and without oral contrast agents; 3) selecting and abstracting data from relevant studies; 4) evaluating and synthesising the literature; and 5) translating results for clinical/operational decision making. Findings of low- to moderate-quality evidence across outcomes, combined with operational and resource data, resulted in a decision not to implement the practice.

Lessons for Guideline Developers, Adaptors, Implementers, and/or Users Coupled with expert input from knowledgeable clinicians and stakeholders, rapid evidence reviews can be critical to shaping evidence-based guidance in Emergency Department settings.

\section{DO MODELS OF RAPID GUIDELINE UPDATES FIT WITHIN THE CURRENT AUSTRALIAN GUIDELINE STANDARDS? AN EXAMPLE FROM THE NATIONAL STROKE FOUNDATION CLINICAL GUIDELINES}

K Hill, L Wright. National Stroke Foundation, Melbourne, Australia

\section{0:1136/bmjas-2013-002293.65}

Background Clinical guideline recommendations are developed to assist health professionals to make evidence-based decisions. This is reliant on having the most up-to-date evidence available. The current Australian National Health and Medical Research Council (NHMRC) standards require guidelines to be updated within five years. An online process which provides transparency and enables timely changes/updates, such as the wiki platform developed and used by the Cancer Council of Australia (CCA), would provide a more useful platform to update guidelines but is currently not considered within the NHMRC standards.

Objectives To evaluate if using the CCA wiki platform to update stroke guidelines would meet NHMRC standards.

Methods We reviewed a potential CCA wiki platform ("wiki") model against the existing NHMRC standards (2011) to determine compatibility and identify where changes to the wiki model might be required.

Results The processes utilising the wiki were methodologically robust and were deemed to comply with $45 / 50$ of the mandatory elements of the NHMRC standards with minor changes needed to comply with the other five elements. Difficulties arise 